\title{
Magnetic-Field-Enhanced Incommensurate Magnetic Order in the Underdoped High-Temperature Superconductor $\mathrm{YBa}_{2} \mathrm{Cu}_{3} \mathrm{O}_{6.45}$
}

\author{
D. Haug, ${ }^{1}$ V. Hinkov, ${ }^{1, *}$ A. Suchaneck, ${ }^{1}$ D. S. Inosov, ${ }^{1}$ N. B. Christensen,${ }^{2,3,4}$ Ch. Niedermayer, ${ }^{2}$ P. Bourges, ${ }^{5}$ Y. Sidis, ${ }^{5}$ \\ J. T. Park, ${ }^{1}$ A. Ivanov, ${ }^{6}$ C. T. Lin, ${ }^{1}$ J. Mesot,,${ }^{2,7}$ and B. Keimer ${ }^{1}$ \\ ${ }^{1}$ Max-Planck-Institut für Festkörperforschung, Heisenbergstraße 1, D-70569 Stuttgart, Germany \\ ${ }^{2}$ Laboratory for Neutron Scattering, ETH Zürich \& Paul Scherrer Institut, CH-5232 Villigen, Switzerland \\ ${ }^{3}$ Materials Research Division, Risф DTU, Technical University of Denmark, DK-4000 Roskilde, Denmark \\ ${ }^{4}$ Nano-Science Center, Niels Bohr Institute, University of Copenhagen, DK-2100 Copenhagen, Denmark \\ ${ }^{5}$ Laboratoire Léon Brillouin, CEA-CNRS, CEA-Saclay, F-91191 Gif-sûr-Yvette, France \\ ${ }^{6}$ Institut Laue-Langevin, 6 Rue Jules Horowitz, F-38042 Grenoble Cedex 9, France \\ ${ }^{7}$ Institut de Physique de la Matière Complexe, EPFL, CH-1015 Lausanne, Switzerland
}

(Received 21 January 2009; published 29 June 2009)

\begin{abstract}
We present a neutron-scattering study of the static and dynamic spin correlations in the underdoped high-temperature superconductor $\mathrm{YBa}_{2} \mathrm{Cu}_{3} \mathrm{O}_{6.45}$ in magnetic fields up to $15 \mathrm{~T}$. The field strongly enhances static incommensurate magnetic order at low temperatures and induces a spectral-weight shift in the magnetic-excitation spectrum. A reconstruction of the Fermi surface driven by the field-enhanced magnetic superstructure may thus be responsible for the unusual Fermi surface topology revealed by recent quantum-oscillation experiments.
\end{abstract}

DOI: 10.1103/PhysRevLett.103.017001

The Landau theory, which treats correlated electron systems as a liquid of weakly interacting quasiparticles, is one of the central tenets underlying our current microscopic understanding of metals. For a long time, the existence of well-defined Landau quasiparticles in underdoped hightemperature superconductors had been called into question, based in part on the unusual thermodynamic and transport properties of these materials. Recently, however, the discovery of quantum oscillations in $\mathrm{YBa}_{2} \mathrm{Cu}_{3} \mathrm{O}_{6.5}$ (Refs. [1-3]) and its close cousin $\mathrm{YBa}_{2} \mathrm{Cu}_{4} \mathrm{O}_{8}$ (Ref. [4]), the two underdoped cuprates least affected by disorder due to randomly placed dopant atoms, has confirmed the presence of coherent fermionic quasiparticles.

While resolving a major open question in research on high-temperature superconductivity, the quantumoscillation experiments have generated a new set of puzzles. The observed oscillation frequencies, combined with the negative sign of the low-temperature Hall coefficient, are indicative of small electronlike Fermi surface pockets. A straightforward electron count then shows that a complex Fermi surface comprising both electron and hole pockets is required to satisfy Luttinger's theorem. Very recent quantum-oscillation data have indeed provided evidence of the hole pockets inferred from these arguments [5]. However, neither angle-resolved photoemission spectroscopy (ARPES) data on $\mathrm{YBa}_{2} \mathrm{Cu}_{3} \mathrm{O}_{6+y}$ [6,7] (or any other hole-doped high-temperature superconductor) nor $a b$ initio band structure calculations for $\mathrm{YBa}_{2} \mathrm{Cu}_{3} \mathrm{O}_{6.5}$ and $\mathrm{YBa}_{2} \mathrm{Cu}_{4} \mathrm{O}_{8}$ (Refs. [8,9]) show clear signs of a Fermi surface topology comprised of small electron and hole pockets.

Keeping in mind that quantum-oscillation experiments require magnetic fields $H \sim 50 \mathrm{~T}$, above the upper critical
PACS numbers: 74.25.Ha, 71.18.+y, 74.72.Bk, 78.70.Nx

field for superconductivity, and that ARPES measurements can be performed only for $H=0$, a Fermi surface reconstruction due to a magnetic-field-induced superstructure offers a possible solution to this puzzle. Indeed, fieldinduced commensurate [10] and incommensurate [11] spin modulations as well as striped [12] and $d$-densitywave [13] states have been invoked as explanations of the small Fermi surface pockets. We use elastic and inelastic neutron scattering to demonstrate that $\mathrm{YBa}_{2} \mathrm{Cu}_{3} \mathrm{O}_{6+y}$ crystals with $y=0.45$ (corresponding to doping levels close to those studied in the quantum-oscillation experiments) develop robust incommensurate magnetic order in high magnetic fields, thus providing strong experimental support for one of the proposed scenarios as well as a perspective for a unified description of the spin correlations and fermiology in the underdoped cuprates.

The neutron-scattering experiments were performed on the same array of untwinned $\mathrm{YBa}_{2} \mathrm{Cu}_{3} \mathrm{O}_{6.45}$ single crystals $\left(T_{c}=35 \mathrm{~K}\right)$ recently shown to exhibit liquid-crystal-like [14] incommensurate spin fluctuations (with a propagation direction selected by the small orthorhombic distortion of the crystal structure) below $\sim 150 \mathrm{~K}$ for $H=0$, while muon-spin-rotation $(\mu \mathrm{SR})$ experiments show static magnetic order only below $\sim 2 \mathrm{~K}$ (Ref. [15]). The array consists of $\sim 100$ almost twin-free $\mathrm{YBa}_{2} \mathrm{Cu}_{3} \mathrm{O}_{6.45}$ single crystals with a total mass of $\sim 2.0 \mathrm{~g}$ and a majority twin domain population of $>92 \%$. The crystals were individually characterized by magnetometry and have a transition temperature of $T_{c}=35 \mathrm{~K}$ with a transition width of $2-3 \mathrm{~K}$. The lattice parameters $a=3.8388 \AA, b=3.8747 \AA$, and $c=$ $11.761 \AA$ correspond to a hole doping level $p_{h}=0.085 \pm$ 0.01 per $\mathrm{Cu}$ ion [16]. Further details about the sample and the detwinning procedure are given in Refs. $[15,17]$. 
The experiments were carried out at the triple-axis spectrometer IN14 at the Institut Laue-Langevin. Pyrolytic graphite crystals, set for the (002) reflection, were used to monochromate and analyze the neutron beam. The final wave vector was fixed to $k_{f}=1.5 \AA^{-1}$. No collimation was used to maximize the neutron flux, and a beryllium filter extinguished higher-order contaminations of the neutron beam.

The sample was mounted in a $15-\mathrm{T}$ vertical-field cryomagnet. The scattering plane was spanned by the vectors $\left(\begin{array}{lll}1 & 0 & 0\end{array}\right)$ and $\left(\begin{array}{lll}0 & 1 & 2\end{array}\right)$. Throughout this Letter, the wave vector $\mathbf{Q}=\left(Q_{H}, Q_{K}, Q_{L}\right)$ is quoted in r.l.u., i.e., in units of the reciprocal lattice vectors $\mathbf{a}^{*}, \mathbf{b}^{*}$, and $\mathbf{c}^{*}\left(a^{*}=2 \pi / a\right.$, etc.). The scattering geometry implies an angle of $\sim 33^{\circ}$ between $\mathbf{H}$ and $\mathbf{c}$. For the field-dependent experiments, the external magnetic field was thus applied mainly perpendicular to the $\mathrm{CuO}_{2}$ planes. All scans for $H \neq 0$ were performed after field cooling.

We first focus on the magnetic response obtained by setting the neutron spectrometer for energy transfer $E=0$. In agreement with previous data on $\mathrm{YBa}_{2} \mathrm{Cu}_{3} \mathrm{O}_{6.45}$ (Ref. [15]), scans taken at temperatures below $\sim 30 \mathrm{~K}$ for $H=0$ in this configuration and shown in Fig. 1(a) exhibit a small incommensurate signal with amplitude $\sim 0.05 \mu_{B}$ per planar $\mathrm{Cu}$ site at $T=2 \mathrm{~K}$, estimated from $\mu$ SR measurements on the same sample [15]. The finite energy resolution $\Delta E \sim 0.2 \mathrm{meV}$ of the instrument implies that
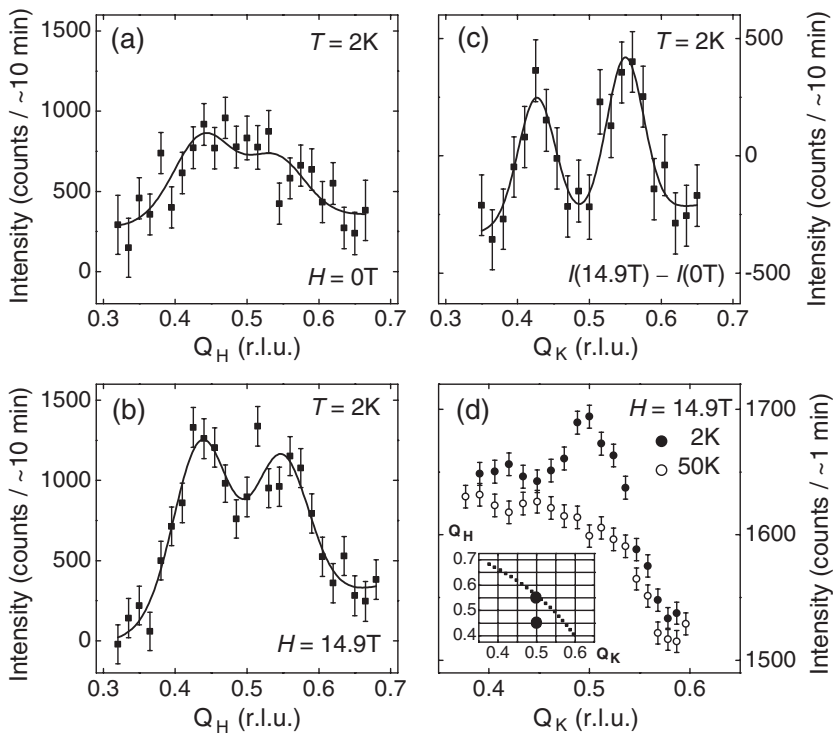

FIG. 1. (a)-(c) Elastic scans along $Q_{H}$ at fixed $Q_{K}=0.5$ and $Q_{L}=1$. The lines are results of two-Gaussian fits to the data. Panels (a) and (b) compare the incommensurate spin modulation at $H=0$ and at $H=14.9 \mathrm{~T}$ at low temperatures $(T=2 \mathrm{~K})$. The background at $T=50 \mathrm{~K}$ was subtracted. Panel (c) visualizes the effect of applying $H=14.9 \mathrm{~T}$ at $T=2 \mathrm{~K}$ by subtracting the intensity at $H=0$ from the intensity at $H=14.9$ T. (d) Raw (uncorrected) rocking scan profile at $H=14.9 \mathrm{~T}$ through the incommensurate peak at $(0.56,0.5,1)$ along the reciprocal-space trajectory indicated in the inset. Closed circles indicate the intensity at $2 \mathrm{~K}$ and open circles the intensity at $50 \mathrm{~K}$. static order as well as slow fluctuations (with energies less than $\Delta E$ ) contribute to this response. However, $\mu \mathrm{SR}$ experiments show that at temperatures around $2 \mathrm{~K}$ the characteristic energy scale for fluctuations of the electron spin system is in the $\mu \mathrm{eV}$ range. Since this is well below the characteristic energy scale $\hbar \omega_{c} \sim 1 \mathrm{meV}$ for cyclotron motion of the electrons at $H \sim 50 \mathrm{~T}[1,3]$, the $E=0$ data can be regarded as a manifestation of static incommensurate magnetic order when discussing implications for the quantum-oscillation data.

Corresponding elastic data at the highest available field $H=14.9 \mathrm{~T}$ demonstrate that the ordered moment increases strongly with magnetic field [Figs. 1(b) and 1(c)]. In order to obtain a quantitative description of the evolution of the incommensurate spin modulation with $H$, the data were fitted to Gaussian profiles (lines in Fig. 1). Neither the resolution-corrected peak width $(0.09 \pm 0.01$ r.l.u. full width at half maximum) nor the incommensurability $(0.049 \pm 0.003$ at $H=0,0.055 \pm 0.003$ r.l.u. at $H=$ $14.9 \mathrm{~T}$ ) vary significantly with $H$. However, the integrated intensity of the elastic peak extracted from these fits increases continuously with $H$ (Fig. 2). At $H=14.9$ T, it is about a factor of 2 larger than the zero-field intensity.

The total moment sum rule for the scattering function [18] stipulates that the spectral weight integrated over all energies and momenta is conserved. This implies that the amplitude of the inelastic response has to decrease in order to compensate for the spectral weight accumulated in the elastic peak. We have therefore studied the effect of the external magnetic field on the low-energy spin excitations. The results are summarized in Fig. 3. For an excitation energy $E=3 \mathrm{meV}$, the spin fluctuation intensity decreases appreciably at $H=14.9 \mathrm{~T}$, while the shape of the scattering profile remains unaffected [Fig. 3(a)]. Surprisingly, the suppression with field is most pronounced around 3-4 meV [Fig. 3(b)]; excitations at lower $E$ are much less affected by the external field. The detailed

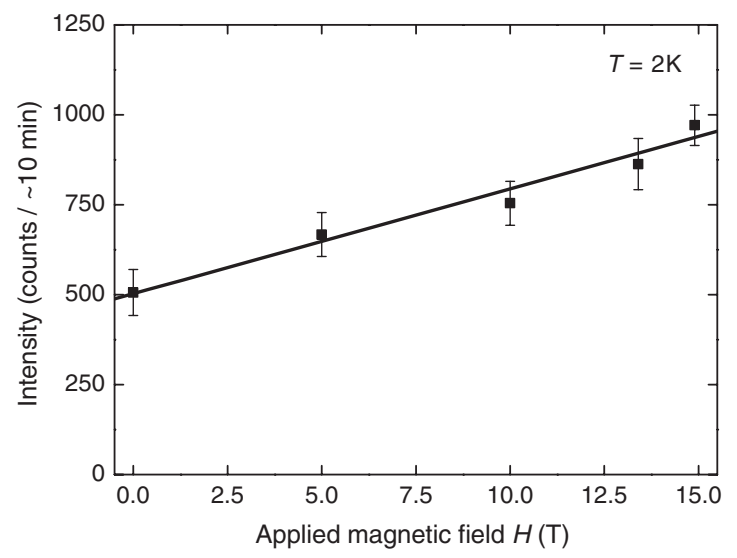

FIG. 2. Dependence of the elastic peak intensity at the incommensurate position $(0.56,0.5,1)$ on the external field at $T=2 \mathrm{~K}$. The data were extracted from the scans shown in Fig. 1 and scans similar to those. The line is the result of a linear fit to the data points. 
quantitative verification of the sum rule is generally complicated for technical reasons restricting the energy range which can be studied at a given spectrometer [19] and in cuprates in addition by the low magnetic intensity [20-22]. Nevertheless, a comparison of the integrated intensities provides valuable insight: Our data indicate that the spectral-weight reduction in the energy range covered by our experiment $(E<4 \mathrm{meV}$ [19]) accounts for roughly half of the gain in elastic intensity with increasing $H$. Figure 3(b) indicates that at least part of the remaining spectral weight originates from a range of a few $\mathrm{meV}$ immediately above our upper limit of $4 \mathrm{meV}$, while we cannot exclude the possibility that some intensity might come from even higher energies.

Previous experiments on the $\mathrm{La}_{2-x} \mathrm{Sr}_{x} \mathrm{CuO}_{4}$ family of high-temperature superconductors have provided ample evidence of field-induced or field-enhanced incommensurate magnetic order [22-28]. A field enhancement of the magnetic order has also been reported for some electrondoped cuprates [29], but the magnetic correlations in this family are commensurate (with a propagation vector identical to the undoped antiferromagnet) at all doping levels. Our observation now indicates that field amplification of incommensurate magnetic order is a universal property of the hole-doped cuprates.

However, we also point out some material-specific aspects of the magnetic phase diagram. First, we note that saturation behavior of the elastic intensity at high field is observed in $\mathrm{La}_{2-x} \mathrm{Sr}_{x} \mathrm{CuO}_{4}$ when the amplitude of the ordered moment reaches $\sim 0.2 \mu_{B}$ [25]. As the ordered moment at the highest field covered by our experiment, $\sim 0.07 \mu_{B}$, is substantially lower, the absence of saturation effects is not surprising. The field-induced amplification of the peak intensity at $E=0$ also generally agrees with theoretical work on incommensurate spin-density wave order competing with $d$-wave superconductivity [30]. Evidence for the theoretically predicted logarithmic corrections to the leading linear $H$ dependence is, however, not apparent in the data shown in Fig. 2.

Second, most of the field-dependent experiments on $\mathrm{La}_{2-x} \mathrm{Sr}_{x} \mathrm{CuO}_{4}$ were performed at doping levels around $1 / 8$ hole per $\mathrm{Cu}$ atom. The doping level of our $\mathrm{YBa}_{2} \mathrm{Cu}_{3} \mathrm{O}_{6.45}$ is significantly lower $(0.085$ holes per planar $\mathrm{Cu}$ ), and the incommensurability of the magnetic response is about a factor of 2 smaller than that of $\mathrm{La}_{2-x} \mathrm{Sr}_{x} \mathrm{CuO}_{4}$ at comparable doping levels.

Unlike the findings at $E=0$, the field-induced response of the low-energy spin excitations in $\mathrm{YBa}_{2} \mathrm{Cu}_{3} \mathrm{O}_{6.45}$ is quite different from corresponding observations in $\mathrm{La}_{2-x} \mathrm{Sr}_{x} \mathrm{CuO}_{4}$-in particular, our data bear no indication of a spin gap closing with field (Fig. 3). To a large extent, we attribute this to the different hole doping: It is sufficiently high in the investigated $\mathrm{La}_{2-x} \mathrm{Sr}_{x} \mathrm{CuO}_{4}$ samples as to result in a pronounced superconducting spin gap [21$23,31]$. In contrast, our sample develops no such gap below $T_{c}$ in the first place [15] and exhibits a robust elastic peak already at $H=0$. Such a phenomenology is established in
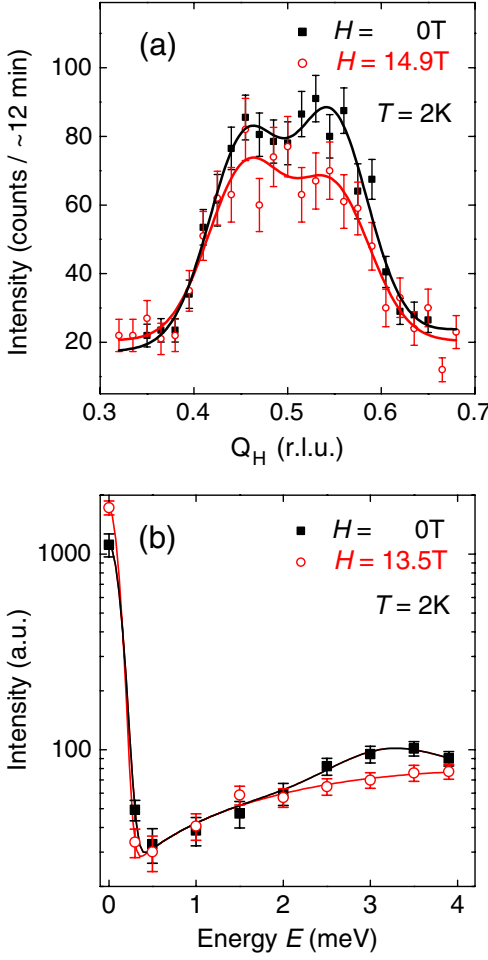

FIG. 3 (color online). (a) Inelastic scans at $E=3 \mathrm{meV}$ and $T=2 \mathrm{~K}$ along $Q_{H}$ at fixed $Q_{K}=0.5$ and $Q_{L}=1$. Black squares indicate the raw scattering intensity at $H=0$ and open circles the intensity at $H=14.9 \mathrm{~T}$. The lines represent the results of two-Gaussian fits to the data. (b) Energy dependence of the magnetic peak intensity at the incommensurate position $(0.56,0.5,1)$ in zero field (closed squares) and in $H=$ 13.5 $\mathrm{T}$ (open circles), corrected for the background. Note the logarithmic intensity scale.

the $\mathrm{La}_{2-x} \mathrm{Sr}_{x} \mathrm{CuO}_{4}$ samples only at elevated fields $H$ (e.g., in Ref. [23]). The main effect of further increasing $H$ is an increase of the elastic intensity, in analogy to what we observe in $\mathrm{YBa}_{2} \mathrm{Cu}_{3} \mathrm{O}_{6.45}$.

While the spectral-weight reduction around 3-4 meV (Fig. 3) is reminiscent of the field-induced suppression of the intensity of the "resonance mode" in more highly doped $\mathrm{YBa}_{2} \mathrm{Cu}_{3} \mathrm{O}_{6.6}$ [32], attributed to a destabilization of the superconducting state through orbital depairing, the close proximity of our sample to the antiferromagnetic insulating state casts doubts on the applicability of such concepts at our low doping level. It is thus interesting to compare our data to the field-induced response of an undoped Heisenberg antiferromagnet with planar exchange anisotropy [33]. In this system, a field applied perpendicularly to the planes hardens magnon excitations polarized out of the plane while leaving the gapless in-plane excitations unaffected. Spin polarization analysis of the inelastic neutron intensity in the presence of a magnetic field is required to assess the validity of this concept.

In summary, we have shown that an external magnetic field strongly enhances static incommensurate magnetic order and induces a spectral-weight rearrangement in the 
magnetic-excitation spectrum of $\mathrm{YBa}_{2} \mathrm{Cu}_{3} \mathrm{O}_{6.45}$. Based on previous studies of $\mathrm{La}_{2-x} \mathrm{Sr}_{x} \mathrm{CuO}_{4}$ [22-28], it is reasonable to expect a similar effect at the slightly larger doping level of $\mathrm{YBa}_{2} \mathrm{Cu}_{3} \mathrm{O}_{6.5}$, where the quantum-oscillation experiments were performed. On general grounds, the fieldenhanced magnetic superstructure is expected to drive a reconstruction of the Fermi surface from the large hole barrels predicted by band structure calculations towards a more complex topology comprised of small electron and hole pockets. In agreement with this argument, the multisheet Fermi surface observed in electron-doped hightemperature superconductors is now generally attributed to the influence of commensurate antiferromagnetism $[34,35]$, which has been independently observed by neutron diffraction [29]. Recent ARPES experiments on Ndand Eu-substituted $\mathrm{La}_{2-x} \mathrm{Sr}_{x} \mathrm{CuO}_{4}$ have also revealed Fermi surface reconstructions possibly associated with stripe order [36,37]. Based on an extrapolation of the intensity of the incommensurate elastic peak of $\mathrm{YBa}_{2} \mathrm{Cu}_{3} \mathrm{O}_{6.45}$ to $50 \mathrm{~T}$, where quantum oscillations are observed to set in, we estimate an ordered moment of $\sim 0.13 \mu_{B}$, which is comparable to the value observed in the fully established stripe-ordered state in $\mathrm{Nd}$-doped $\mathrm{La}_{2-x} \mathrm{Sr}_{x} \mathrm{CuO}_{4}$ [28] and the typical ordered moments in superconducting electron-doped cuprates [29].

It is therefore likely that the incommensurate magnetic order we have found strongly influences the Fermi surface geometry determined by the high-field quantum-oscillation experiments. Theoretical calculations of the Fermi surface geometry in the presence of stripe order with commensurate in-plane wave vector $1 / 8$, which were inspired by experiments in $\mathrm{La}_{2-x} \mathrm{Sr}_{x} \mathrm{CuO}_{4}$, support this conclusion and demonstrate that this mechanism can yield quantumoscillation frequencies of the correct order of magnitude [12]. Our experiments now show that a generalization of this work to incommensurate magnetic order with substantially smaller wave vectors is required to obtain a quantitative description of the quantum-oscillation data. In closing, we note that our experiments probe only spin (and not charge) degrees of freedom and do not discriminate between longitudinal (stripe) [12] and transverse (spiral) [38] modulations of the spin system. It therefore appears worthwhile to explore the possible influence of both types of order on the physical properties of $\mathrm{YBa}_{2} \mathrm{Cu}_{3} \mathrm{O}_{6+y}$ at high magnetic fields.

We thank O. Sushkov and C. Bernhard for stimulating discussions, A. Hiess and M. Böhm for support during the experiment at IN14, S. Lacher, B. Baum, and H. Wendel for their help in sample preparation, C. Busch and H. Bender for technical support, and R. Dinnebier for determining the lattice parameters using $\mathrm{X}$-ray powder diffraction. We acknowledge financial support by the DFG (FOR538) and DANSCATT.

*V.Hinkov@fkf.mpg.de
[1] N. Doiron-Leyraud et al., Nature (London) 447, 565 (2007).

[2] D. LeBoeuf et al., Nature (London) 450, 533 (2007).

[3] C. Jaudet et al., Phys. Rev. Lett. 100, 187005 (2008).

[4] E. A. Yelland et al., Phys. Rev. Lett. 100, 047003 (2008); A.F. Bangura et al., Phys. Rev. Lett. 100, 047004 (2008).

[5] S. E. Sebastian et al., Nature (London) 454, 200 (2008).

[6] M. A. Hossain et al., Nature Phys. 4, 527 (2008).

[7] V.B. Zabolotnyy et al., Phys. Rev. B 76, 064519 (2007).

[8] A. Carrington and E. A. Yelland, Phys. Rev. B 76, 140508 (R) (2007).

[9] I. S. Elfimov, G. A. Sawatzky, and A. Damascelli, Phys. Rev. B 77, 060504(R) (2008).

[10] W.-Q. Chen, K.-Y. Yang, T. M. Rice, and F.C. Zhang, Europhys. Lett. 82, 17004 (2008).

[11] N. Harrison, Phys. Rev. Lett. 102, 206405 (2009).

[12] A. J. Millis and M. R. Norman, Phys. Rev. B 76, 220503 (R) (2007).

[13] S. Chakravarty and H. Y. Kee, Proc. Natl. Acad. Sci. U.S.A. 105, 8835 (2008)

[14] S. A. Kivelson, E. Fradkin, and V. J. Emery, Nature (London) 393, 550 (1998).

[15] V. Hinkov et al., Science 319, 597 (2008).

[16] R. Liang, D. A. Bonn, and W. N. Hardy, Phys. Rev. B 73, 180505(R) (2006).

[17] V. Hinkov et al., Nature Phys. 3, 780 (2007).

[18] J. Lorenzana, G. Seibold, and R. Coldea, Phys. Rev. B 72, 224511 (2005).

[19] In our experiment, to be able to study both the elastic peak and the low-energy spin excitations, we had to use a cold triple-axis spectrometer. This, in conjunction with the bulky 15-T cryomagnet, limited the energy range for geometrical reasons.

[20] H. F. Fong, B. Keimer, D. Reznik, D. L.Milius, and I. A. Aksay, Phys. Rev. B 54, 6708 (1996).

[21] J. M. Tranquada et al., Phys. Rev. B 69, 174507 (2004).

[22] J. Chang et al., Phys. Rev. Lett. 98, 077004 (2007).

[23] J. Chang et al., Phys. Rev. Lett. 102, 177006 (2009).

[24] S. Katano, M. Sato, K. Yamada, T. Suzuki, and T. Fukase, Phys. Rev. B 62, R14 677 (2000).

[25] B. Lake et al., Nature (London) 415, 299 (2002).

[26] B. Khaykovich et al., Phys. Rev. B 66, 014528 (2002).

[27] B. Khaykovich et al., Phys. Rev. B 71, 220508(R) (2005).

[28] J. Chang et al., Phys. Rev. B 78, 104525 (2008).

[29] H. J. Kang et al., Phys. Rev. B 71, 214512 (2005).

[30] E. Demler, S. Sachdev, and Y. Zhang, Phys. Rev. Lett. 87, 067202 (2001).

[31] B. Lake et al., Science 291, 1759 (2001).

[32] P.C. Dai, H.A. Mook, G. Aeppli, S. M. Hayden, and F. Doğan, Nature (London) 406, 965 (2000).

[33] J. M. Tranquada, G. Shirane, B. Keimer, S. Shamoto, and M. Sato, Phys. Rev. B 40, 4503 (1989).

[34] S. R. Park et al., Phys. Rev. B 75, 060501(R) (2007).

[35] N. Harrison, R. D. McDonald, and J. Singleton, Phys. Rev. Lett. 99, 206406 (2007).

[36] J. Chang et al., New J. Phys. 10, 103016 (2008).

[37] V.B. Zabolotnyy et al., Europhys. Lett. 86, 47005 (2009).

[38] A. I. Milstein and O. P. Sushkov, Phys. Rev. B 78, 014501 (2008). 